\title{
Bolo de "chocolate" produzido com pó de cupuaçu e kefir
}

\author{
Mauricio Sergio Esteller, Orlando Zancanaro Júnior, Suzana Caetano da Silva Lannes*
}

${ }^{1}$ Departamento de Tecnologia Bioquímico-Farmacêutica, Faculdade de Ciências Farmacêuticas, Universidade de São Paulo

*Correspondência:

S. C. S. Lannes

Departamento de Tecnologia

Bioquímico-Farmacêutica

Faculdade de Ciências

Farmacêuticas - USP

Av. Prof. Lineu Prestes, 580 - Bloco 16 05508-900 - São Paulo, SP - Brasil

E-mail: scslan@usp.br
Nos últimos anos vem ocorrendo a valorização dos recursos e produtos naturais. Cresce em todo o mundo a preocupação com a conservação do meio ambiente e a valorização dos recursos da flora e fauna. É forte a demanda por tecnologias de processo, de produto e de aplicação. Neste estudo foi utilizada formulação padronizada para bolo tipo inglês, substituindo cacau em pó por cupuaçu em pó e com adição de kefir, mantendo-se o total de sólidos. O tempo e a temperatura de assamento foram fixados em 50 minutos e $180{ }^{\circ} \mathrm{C}$. Foram avaliados a porosidade do miolo, o perfil de textura, cor, umidade, volume específico e densidade da massa. Os resultados mostraram que a adição de pó de cupuaçu não altera as características do bolo padrão elaborado com cacau e que a adição de kefir modifica a textura e a porosidade do miolo.

\section{INTRODUÇÃO}

Tanto da polpa quanto da semente do cupuaçu (Theobroma grandiflorum) vários subprodutos podem ser obtidos e estudados. "Chocolate" em pó pode ser obtido a partir da moagem da torta desengordurada de cupuaçu, misturados com outros ingredientes. A matéria-prima para a produção da pasta de cupuaçu é constituída por semente de cupuaçu, após fermentação, secagem e moagem (Lannes et al., 2003).

Kefir é uma bebida originária do Cáucaso, produzida pela ação de bactérias lácticas, acéticas e leveduras no leite, também conhecido por kephir, kiaphur, kefyr, képhir, kéfer, knapon, kepi e kippe. Os grânulos de kefir são constituídos por $66 \%$ de bacilli, $16 \%$ de streptococci e $18 \%$ de leveduras ou 890 a $900 \mathrm{~g} / \mathrm{kg}$ de água, $2 \mathrm{~g} / \mathrm{kg}$ de gorduras, $30 \mathrm{~g} / \mathrm{kg}$ de proteínas, $60 \mathrm{~g} / \mathrm{kg}$ de açúcares e $7 \mathrm{~g} / \mathrm{kg}$ de cinzas (Farnworth, 2003).

\author{
Unitermos \\ - Cupuaçu \\ - Kefir \\ - Bolos \\ - Textura \\ - Análise de Imagens \\ - Cor
}

Massas de bolo são constituídas por um sistema bifásico: gorduras + outros ingredientes da mistura. A fase gordurosa apresenta-se dispersa de forma irregular com partículas de forma e tamanho variados. Internamente às partículas de gordura são encontradas inúmeras bolhas de ar incorporadas durante o processo de mistura e batimento. A formação de estrutura uniforme dos alvéolos é de grande importância para a qualidade de massas assadas. Uma emulsão estável permite boa evaporação da água e expansão do gás carbônico liberado, resultado das reações do fermento. A adição de cacau, fibras, amidos e frutas secas picadas ou moídas é crítica, pois pode deixar o produto com aparência "embatumada", isto é, maior crescimento na parte superior e massa úmida e compacta na parte inferior. O batimento (formação de creme) e controle do overrun (quantidade de ar incorporada) estão diretamente relacionados à qualidade da massa (Jooste, 1951). 
A qualidade de bolos pode ser avaliada por meios instrumentais e sensoriais. O procedimento para determinação da firmeza por métodos instrumentais e demais parâmetros de textura no bolo, consiste em fatiar as amostras, submetê-las à compressão em texturômetro mecânico e analisar a curva força-tempo resultante (Esteller et al., 2004). A microestrutura determina em larga extensão as propriedades reológicas e sensoriais. A cor é um parâmetro crítico em produtos forneados. Bolos com crosta muito claras ou muito escuras estão associados a falhas no processo e formulação (Esteller et al., 2005, 2006a).

O objetivo deste trabalho é produzir e estudar os parâmetros de qualidade em bolo de "chocolate" produzido com diferentes quantidades de pó de cupuaçu e kefir, utilizado como substituto do fermento industrial tradicional, e compará-lo com um controle com adição de pó de cacau tradicional e sem kefir.

\section{MATERIAL E MÉTODOS}

\section{Material}

No preparo das massas foram utilizadas farinha de trigo (Sol), açúcar refinado (União), margarina culinária (Primor), emulsificante Glintex (Lida), ovos (CAC), kefir (USP), cupuaçu pó (USP), cacau pó (Nestlé), leite em pó integral (Nestlé), sal refinado (Cisne), aroma natural de baunilha em pó (Duas Rodas), aroma natural de chocolate em pó (Duas Rodas) e fermento químico (Royal). A massa foi preparada em batedeira planetária KitchenAid $525 \mathrm{~W} / 120 \mathrm{~V} / 60 \mathrm{~Hz}$ (Brastemp). Os bolos foram assados em forno elétrico tipo

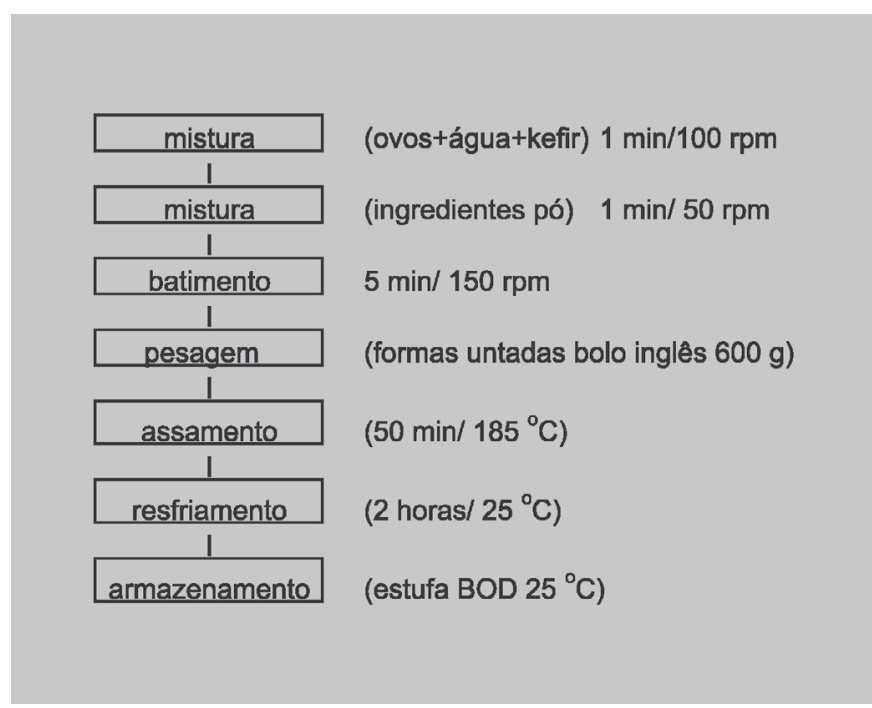

FIGURA 1 - Fluxograma de processo para o preparo da massa de bolo

estacionário 4000W/220V (Flexa de Ouro/SP). As formulações (produzidas em triplicata) e processo empregado são apresentados, respectivamente, na Tabela I e Figura 1.

\section{Métodos}

\section{Porosidade do miolo}

Foram preparadas 8 fatias de cada amostra, com espessura de $25 \mathrm{~mm}$ (desprezando-se as pontas), escaneadas em scanner HP Scanjet 2400, área central do miolo $50 \mathrm{~mm}$ x $50 \mathrm{~mm}, 200 \mathrm{dpi}$, analisadas com o software de imagens Image-Pro Plus 4.5, (Media Cybernetics, Inc., USA). Deter-

TABELA I - Formulações utilizadas para o teste experimental da massa de chocolate

\begin{tabular}{lccccccccc}
\hline Formulação & controle & 1 & 2 & 3 & 4 & 5 & 6 & 7 & 8 \\
Ingredientes (g) & & & & & & & & & \\
\hline Farinha de trigo & 250 & 250 & 250 & 250 & 250 & 250 & 250 & 250 & 250 \\
Açúcar refinado & 250 & 250 & 250 & 250 & 250 & 250 & 250 & 250 & 250 \\
Margarina & 250 & 250 & 250 & 250 & 250 & 250 & 250 & 250 & 250 \\
Emulsificante & 20 & 20 & 20 & 20 & 20 & 20 & 20 & 20 & 20 \\
Ovos & 250 & 250 & 250 & 250 & 250 & 250 & 250 & 250 & 250 \\
Kefir & 0 & 0 & 50 & 100 & 150 & 0 & 50 & 100 & 150 \\
Água & 150 & 150 & 100 & 50 & 0 & 150 & 100 & 50 & 0 \\
Cupuaçu pó & 0 & 25 & 50 & 0 & 25 & 50 & 0 & 25 & 50 \\
Cacau pó & 50 & 25 & 0 & 50 & 25 & 0 & 50 & 25 & 0 \\
Leite pó & 25 & 25 & 25 & 25 & 25 & 25 & 25 & 25 & 25 \\
Sal & 4 & 4 & 4 & 4 & 4 & 4 & 4 & 4 & 4 \\
Aroma baunilha & 2,5 & 2,5 & 2,5 & 2,5 & 2,5 & 2,5 & 2,5 & 2,5 & 2,5 \\
Aroma chocolate & 2,5 & 2,5 & 2,5 & 2,5 & 2,5 & 2,5 & 2,5 & 2,5 & 2,5 \\
Fermento químico & 15 & 15 & 15 & 15 & 15 & 15 & 15 & 15 & 15 \\
\hline
\end{tabular}


minou-se os parâmetros alveolares: área $\left(\mathrm{mm}^{2}\right)$, diâmetro médio $(\mathrm{mm})$ e perímetro $(\mathrm{mm})$.

\section{Textura}

As análises foram realizadas no $2^{\circ}$ dia de armazenamento com 8 fatias $(25 \mathrm{~mm})$ de cada amostra tomando-se os valores médios para cada parâmetro. Foi utilizado o texturômetro TA-XT2 (Stable Micro Systems, UK), com análise de dupla compressão, probe cilíndrico $25 \mathrm{~mm}$ perspex $\mathrm{P} / 25 \mathrm{P}, 2,0 \mathrm{~mm} / \mathrm{s}$, test speed $1,7 \mathrm{~mm} / \mathrm{s}$, trigger type auto, post-test speed $10 \mathrm{~mm} / \mathrm{s}$, distance 6,2 mm, force $10 \mathrm{~g}$, acquisition 200 pps. Parâmetros: Firmeza $(\mathrm{N})$, coesividade, mastigabilidade (N.mm), elasticidade e adesividade (mJ) (Esteller et al., 2004).

\section{Cor}

As amostras tiveram seus valores de reflectância $\left(L^{*}, a^{*}, b^{*}\right)$ registrados no espectrofotômetro HunterLab, modelo UltraScan ${ }^{\mathrm{TM}} \mathrm{XE}$ e sistema CIELAB, com ângulo do observador de $10^{\circ}$ e iluminante D65, $420 \mathrm{~nm}$, ajustado para reflectância. Após calibração, utilizando-se o software Universal 4.10 (Universal Inc., USA), cada fatia de $25 \mathrm{~mm}$ (crosta e miolo), foi colocada com a parte central voltada para a abertura de 1". Cada valor apresentado representa a média entre 8 amostras (Esteller et al., 2006).

\section{Volume, volume específico e densidade}

O volume foi determinado (triplicata) preenchendo-se totalmente, com micro-esferas de polietileno, um copo de vidro liso e transparente com dimensões $7,5 \mathrm{~cm}$ altura x 7,5 cm de diâmetro. Em seguida parte delas foi substituída pela amostra (cubos com aresta de $3 \mathrm{~cm}$ ), completando-se o volume até a borda e nivelamento com auxílio de régua plástica. As esferas remanescentes, correspondendo ao volume final, foram colocadas em cilindro graduado expressando o resultado em $\mathrm{mL}$. As amostras foram pesadas e calculou-se o volume específico $(\mathrm{mL} / \mathrm{g})$ e densidade $(\mathrm{g} / \mathrm{mL})$ (Esteller et al., 2005).

\section{Umidade}

As amostras foram picadas (crosta + miolo), pesando-se cerca de $10 \mathrm{~g}$ em cápsula de alumínio tarada. Os conjuntos (triplicata), foram colocados em estufa a $105^{\circ} \mathrm{C}$ por duas horas. Após desidratação foram mantidos em um dessecador contendo sílica-gel, até atingir a temperatura ambiente, sendo então novamente pesados. O procedimento de secagem e pesagem foi repetido até que um valor constante fosse obtido para cada amostra analisada; o teor de umidade foi calculado pela diferença entre o peso inicial e final das amostras e expresso em porcentagem (Esteller et al., 2006a).

\section{Estatística}

Os dados foram analisados utilizando-se o software Statistica 6.0/2001 (StatSoft, Inc., Tulsa, OK, USA), Tukey HSD 5\% para verificação de variação significativa entre as formulações.

\section{RESULTADOS E DISCUSSÃO}

\section{Porosidade (estrutura alveolar)}

Os valores mínimos para área dos alvéolos $(0,016$ $\left.\mathrm{mm}^{2}\right)$, diâmetro $(0,06 \mathrm{~mm})$ e perímetro $(0,13 \mathrm{~mm})$ para cada formulação de bolo são os mesmos devido ao limite de detecção do software de imagem. As diferenças aparecem para os valores máximos e médios. A quantidade e o volume dos alvéolos estão diretamente relacionados com a formulação e processos utilizados. Massas com batimento insuficiente, má distribuição de ar, não homogêneas e com excesso de fermento tendem a criar, após assados, produtos com alvéolos de grandes dimensões (largos e profundos) (Jooste, 1951).

Observando-se os resultados obtidos, em relação ao controle, ocorreu a "abertura do miolo" ou aumento da porosidade da massa em especial na formulação 8 com adição máxima de kefir ( $150 \mathrm{~g})$, substituindo toda a água da formulação e $50 \mathrm{~g}$ de pó de cupuaçu e, também, substituindo-se todo o cacau, havendo aumento nas dimensões dos alvéolos. Essas mudanças são observadas, também, nas outras formulações: área máxima (189 $\mathrm{mm}^{2}$ controle) para $\left(232-322 \mathrm{~mm}^{2}\right)$; área média $\left(0,31 \mathrm{~mm}^{2}\right)$ para $\left(0,35-0,48 \mathrm{~mm}^{2}\right)$; diâmetro máximo $(21 \mathrm{~mm})$ para $(20-30 \mathrm{~mm})$, diâmetro médio $(0,30 \mathrm{~mm})$ para $(0,28-0,32 \mathrm{~mm})$; perímetro máximo $(148 \mathrm{~mm})$ para $(172-208 \mathrm{~mm})$ e perímetro médio $(1 \mathrm{~mm})$ para $(0,94-$ $1,21 \mathrm{~mm})(\mathrm{p}<0,05)$. Existem poucos trabalhos relatando o uso de kefir em produtos submetidos à cocção. Plesas et al. (2004), descrevem que o uso de grãos de kefir em pães ocasiona a redução do volume $(\mathrm{mL})$, do volume específico $(\mathrm{mL} / \mathrm{g})$, aumenta a acidez da massa e reduzem a umidade no produto final.

\section{Textura}

A dureza ou firmeza do bolo de chocolate, avaliada por métodos instrumentais (texturômetros) é proporcional à força aplicada para ocasionar uma deformação ou rompimento da amostra e está correlacionada com a mordida humana durante a ingestão dos alimen- 
tos (Esteller et al., 2004a). A força máxima avaliada, para alimentos dessa natureza, é dependente da formulação (qualidade da farinha, quantidade de açúcares, gorduras, emulsificantes e ovos), umidade da massa e conservação (tempo de fabricação do produto e embalagem). No processo de batimento da massa, apesar da forte agitação e velocidade das pás, os ingredientes não são solubilizados completamente. Ocorre liberação de gás carbônico antes e durante o forneamento. Durante a cocção ocorre, também, volatilização do vapor d'água da massa e formação de uma matriz complexa de amido gelatinizado e proteínas, que aprisionam o material volátil e formam alvéolos de tamanhos variados. Esses "colchões" de ar, distribuídos de forma não uniforme, podem representar, em uma mesma amostra, variações nos valores de firmeza e influenciar os outros parâmetros de textura. A análise dos resultados de textura apontam para valores baixos de firmeza (ou maior maciez) para o miolo de bolos com bastante umidade, gordura e açúcar, que exercem efeito amaciante na massa. Bolo com tempo excessivo de forneamento, que tenha perdido de alguma forma a umidade (embalagem aberta, por exemplo), apresenta-se como uma massa firme que necessita de maior salivação e mastigação (Esteller et al., 2004a,b).

Nas respostas de textura na Tabela II, a adição de kefir e cupuaçu elevou os valores de firmeza $(3,81 \mathrm{~N}$ controle) para $(3,82-4,68 \mathrm{~N})$. O leite associado ao kefir introduz maior quantidade de proteínas (lactoalbuminas) e, provavelmente, favorece as ligações cruzadas com as grandes moléculas de glúten (glutenina e gliadina), ovoalbumina, amilose e amilopectina ocasionando maior resistência da massa à compressão.

A manutenção da coesividade em derivados de trigo está relacionada principalmente às interações moleculares dos componentes, principalmente pontes de hidrogênio, dissulfeto e ligações cruzadas com a participação de íons metálicos e a mobilidade da água na massa. Durante o período de armazenamento ocorrem mudanças nas ligações (migração de água, cristalização do amido, hidrólise da gordura) levando a um desarranjo gradual da estrutura. Valores baixos de coesividade caracterizam bolos de difícil manipulação e fatiamento porque esfarelam com facilidade.

O açúcar melhora a coesividade. A gordura melhora a maciez (menor firmeza) mas, em excesso, reduz a coesividade. De forma geral massas ricas em gorduras e açúcares são macias, apresentam maior elasticidade e facilitam a mastigação. Excesso torna as massas "podres" (baixa coesividade) e a massa se desagrega com facilidade (Esteller et al., 2004a).

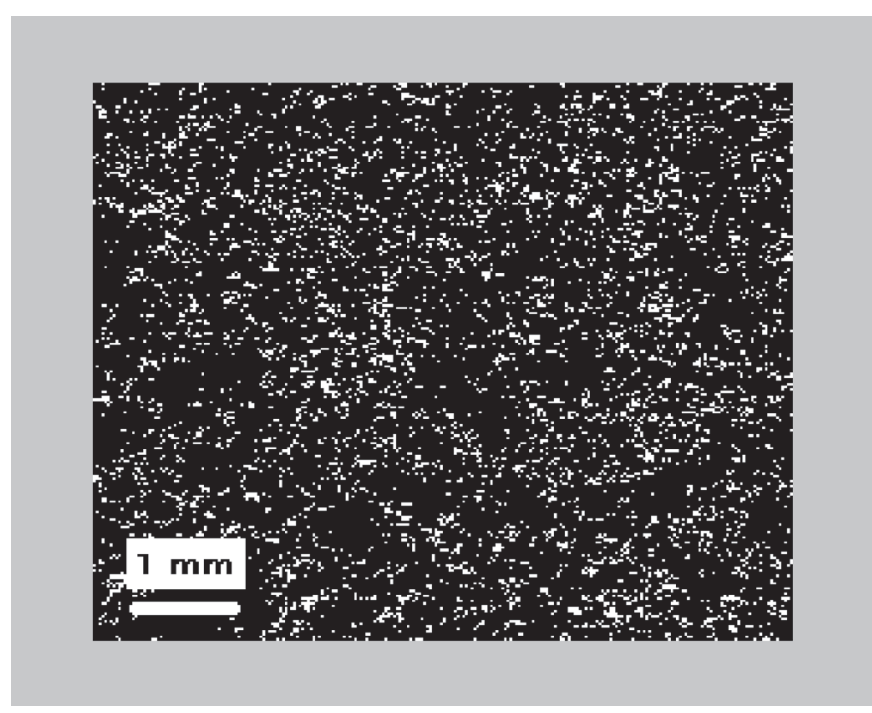

FIGURA 2 - Imagem digital do bolo de "chocolate" $50 \mathrm{~mm}$ x $50 \mathrm{~mm} 200 \mathrm{dpi}$

Nas formulações analisadas, a variação nos ingredientes adicionados e a quantidade parece não influir de forma significativa em perda de coesividade $(0,62$ unidades) no controle e (0,56-0,63 unidades) nas formulações com kefir e pó de cupuaçu, ou redução da elasticidade da massa $(0,74$ unidades) para $(0,67-0,76$ unidades) em relação ao controle. Produtos "envelhecidos" (maior tempo de estocagem), perdem sua elasticidade (Esteller et al., 2004a).

A mastigabilidade é um parâmetro de textura facilmente correlacionado com análise sensorial através de painéis treinados. Amostras com maior teor de fibras ou ressecadas necessitam de maior salivação e número maior de mastigações antes da deglutição. O enrijecimento de massas provoca maior necessidade de trabalho mecânico e movimentação da boca (Esteller et al., 2004a). Os resultados apontam que as massas com adição de cupuaçu e kefir não tiveram a mastigabilidade alterada (1,59-2,19 N.mm) e ficando muito próximos do controle $(1,80$ N.mm $)(p<0,05)$.

São poucas as referências anteriores para avaliação de adesividade em produtos forneados e em particular para bolos. É de se esperar, no entanto, que um aumento na umidade da massa, presença de sacarose e ingredientes com propriedades umectantes, tempo de estocagem e embalagem influenciarão na adesividade avaliada em texturômetros (Esteller et al., 2004a). A interface amostra-probe é bastante sensível às pequenas variações na superfície das amostras. Os valores de adesividade obtidos $(0,001-0,004 \mathrm{~mJ})$ podem ser considerados, no entanto, bastante baixos levando-se em consideração a grande porcentagem de açúcares nas formulações o que poderia tornar a massa bastante pegajosa. 


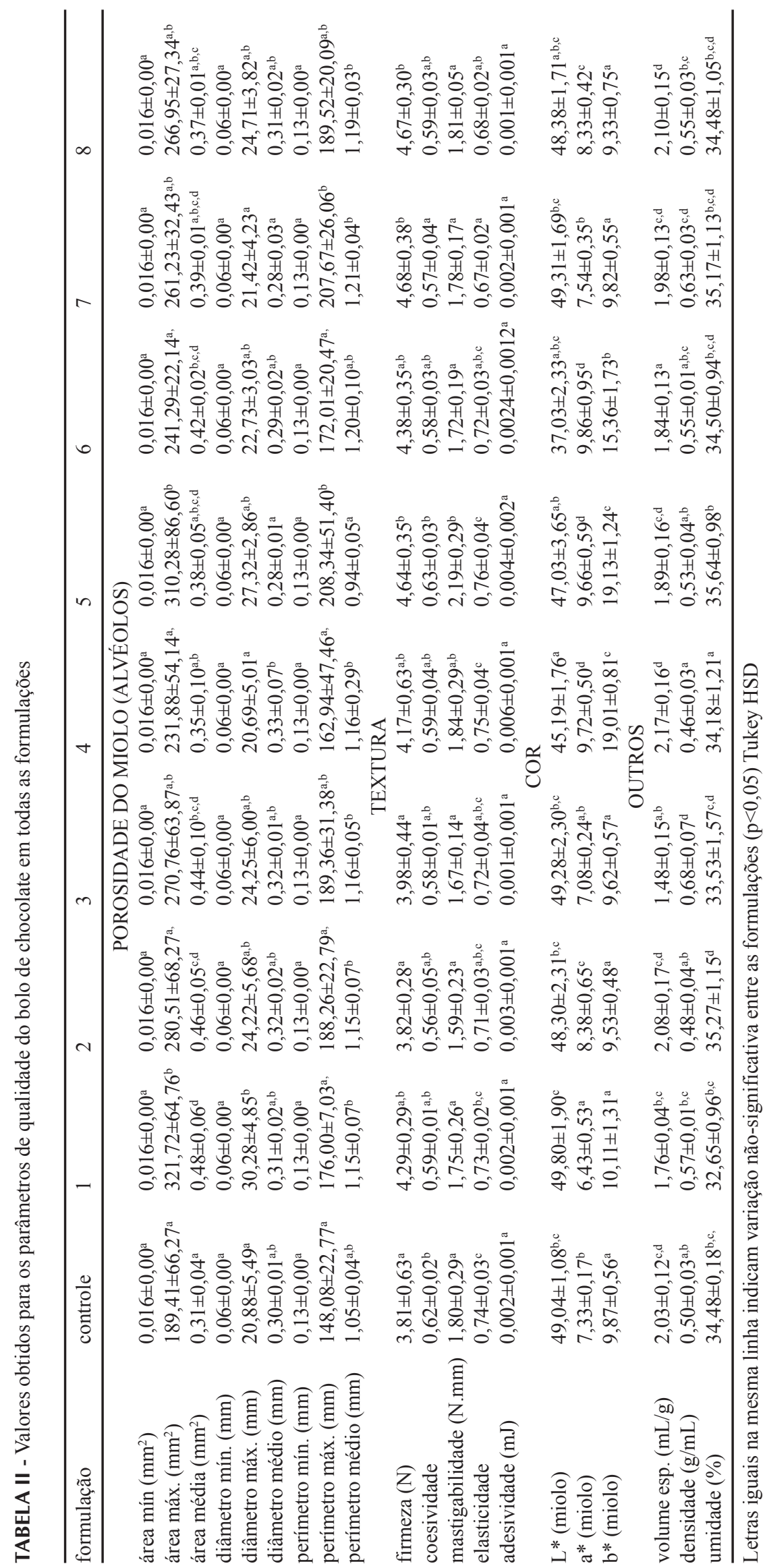




\section{Cor}

Para o assamento do bolo foi favorável uma temperatura maior no "lastro" $\left(200{ }^{\circ} \mathrm{C}\right)$ e menor no "teto" $\left(170^{\circ} \mathrm{C}\right)$, com forno de resistência elétrica "exposta", o que permite melhor acabamento de superfície e abertura da crosta, característica para este tipo de bolo. No centro do miolo a temperatura atinge cerca de $98^{\circ} \mathrm{C}$. A presença de açúcares e ovos na formulação assim como o calor aceleram reações de caramelização e Maillard, levando ao escurecimento progressivo da crosta e miolo, que podem ser desejados ou não (Giese, 2000).

Valores de $L^{*}$ mais altos indicam maior reflectância da luz traduzindo-se em bolo com coloração clara, pobre em açúcares, cacau/cupuaçu, fermento químico inadequado, temperatura baixa e tempo insuficiente de cocção. Maiores valores de a* indicam coloração mais escura (Esteller et al., 2006b).

Valores altos para $b^{*}$ são traduzidos para amostras com forte coloração amarelada ou dourada, que embora "diluída" na coloração castanho escura característica de produtos com derivados de cacau/cupuaçu, podem, ser "filtradas" e aparecem em produtos ricos em proteínas, açúcares redutores e ovos (carotenóides). As observações concordam com Baik et al. (2000) que, na análise de massas de bolo e utilizando o sistema CIELAB, verificam que com o aumento do tempo de forno ocorre aumento no escurecimento da massa, ocasionando redução nos valores de $\mathrm{L}^{*} \mathrm{e}$, proporcionalmente, aumento nos valores de $\mathrm{a}^{*} \mathrm{e} \mathrm{b}^{*}$. Kim et al., (2001) observam que a substituição da fase gordurosa de bolos tipo pão-de-ló, por dextrinas, ocasionam a redução nos valores L*,a*,b*. Na Tabela III estão relacionados os valores de $\mathrm{L}^{*}, \mathrm{a}^{*}, \mathrm{~b}^{*}$ (miolo) de alguns produtos comerciais. Os resultados podem variar bastante em função da formulação e processo aplicados pelos fabricantes.

Comparando-se com o controle (49 unidades), os valores de $\mathrm{L}^{*}$ nas amostras analisadas permaneceu estável (45-50 unidades). Uma coloração mais escura, se desejá- vel, pode ser obtida com a combinação de cacau ou cupuaçu com maior grau de alcalinização. O que concorda com os valores obtidos com produtos comerciais que utilizam este recurso.

\section{Volume específico e densidade}

O volume específico e a densidade mostram claramente a relação entre o teor de sólidos e a fração de ar existente na massa assada. Massas com densidade alta ou volume específico baixo (embatumadas), apresentam aspecto desagradável ao consumidor, associadas com alto teor de umidade, falhas no batimento e cocção, pouca aeração, difícil mastigação, sabor impróprio e baixa conservação. Por isso os bolos necessitam de quantidade adequada de líquidos, fermento químico, emulsificante e aeração adequados. Granulometria mais "aberta" é característica de massas com grande volume (maior fração ar-sólidos) e menor densidade. Os bolos, pelo seu alto teor de líquidos, apresentam-se como produtos macios e aerados, com menor volume específico e maior densidade da massa, quando comparados com outros produtos derivados de trigo como pães e biscoitos (Esteller et al., 2005).

Quando comparado com o controle $(2,03 \mathrm{~mL} / \mathrm{g}) \mathrm{o}$ volume específico das amostras com pó de cupuaçu e kefir apresentaram variações significativas $(p<0,05)$ $(1,48-2,17 \mathrm{~mL} / \mathrm{g})$, o mesmo ocorrendo para a densidade $(0,50 \mathrm{~g} / \mathrm{mL})$ para o controle e $(0,46-0,68 \mathrm{~g} / \mathrm{mL})$ para as demais formulações.

\section{Umidade}

Em bolos industrializados a umidade situa-se em valores próximos a 30\% (Jooste, 1951). Os valores de análise obtidos, descritos na Tabela II estão coerentes com o esperado. A perda e o ganho de umidade vão ocorrer continuamente, de uma região para outra, como forma de equilíbrio dinâmico entre os componentes e o meio (Labuza et al., 1998). Bolos quebrados ou

TABELA III - Valores de L*, $\mathrm{a}^{*}, \mathrm{~b}^{*}$ (miolo) para bolos de chocolate (marcas locais)

\begin{tabular}{lccc}
\hline Produto & $\mathrm{L}^{*}$ & $\mathrm{a}^{*}$ & $\mathrm{~b}^{*}$ \\
\hline Bolo Choc.Carrefour & $46,52 \pm 1,03$ & $6,11 \pm 1,78$ & $7,05 \pm 0,68$ \\
Bolo Choc. Casa Suiça & $43,67 \pm 1,55$ & $5,21 \pm 0,93$ & $5,83 \pm 1,29$ \\
Bolo Choc. Nutrella & $40,78 \pm 1,44$ & $8,34 \pm 0,58$ & $7,45 \pm 1,08$ \\
Bolo Choc. Panco & $46,26 \pm 0,34$ & $6,90 \pm 0,67$ & $8,03 \pm 1,02$ \\
Bolo Choc. Pullman & $45,28 \pm 0,32$ & $5,57 \pm 1,39$ & $6,23 \pm 0,88$ \\
Rocambole Choc.Pullman & $54,62 \pm 2,56$ & $9,05 \pm 0,65$ & $14,40 \pm 1,81$ \\
\hline
\end{tabular}


esfarelando são deixados de lado pelo consumidor no ponto de venda. Umidade em excesso aumenta a atividade microbiana e deixa as massas "grudentas", cabendo ao fabricante, portanto, o controle do teor de umidade incluindo embalagens que protejam o produto da oxidação da gordura e perda de aroma e sabor. Cauvain (1998) relaciona algumas alterações que ocorrem em bolos vinculadas às condições de armazenamento, embalagem, temperatura e umidade relativa como aumento da dureza devido à perda de água para a atmosfera e à migração da água do recheio para a massa, tendência ao esfarelamento devido às alterações na coesividade $\mathrm{e}$ modificações - normalmente perda - no aroma e sabor. A utilização de cupuaçu em pó e kefir não alterou de forma significativa $(\mathrm{p}<0,05)$ a umidade final das amostras em relação ao controle $(34,48 \%)$ e $(32,65-35,64)$ para as demais formulações.

\section{CONCLUSÕES}

A adição de pó de cupuaçu não altera as características do bolo padrão elaborado com cacau, e a adição de kefir modifica a textura e a porosidade do bolo.

A avaliação da porosidade do miolo, por análise de imagens utilizando escaneamento das amostras, contribuiu para uma melhor caracterização desta classe de alimento e desenvolvimento de produtos com textura planejada.

O kefir mostrou-se um produto biotecnológico com potencial para aplicações, também, em produtos forneados e, apesar da sua produção artesanal, pode ser utilizado como agente modificador de textura e paladar.

\section{ABSTRACT}

\section{"Chocolate" pound cake with cupuassu and kefir}

In the last years, natural resources and products have gained the attention of consumers. The concern with the conservation of the environment and the importance of the flora and fauna increases in the whole world. The demand for application, product, and process technologies is strong. In this study, a control chocolate pound cake formula was used changing cocoa powder from the original formula to cupuassu powder and adding kefir, keeping the amounts of total solids. The time and temperature were fixed in 50 minutes and $180^{\circ} \mathrm{C}$. Crumb porosity, texture profile analysis (TPA), color, moisture, specific volume, and dough density were evaluated. The results showed that cupuassu powder can be used as a cocoa replacer in "chocolate" cake production and that kefir changed the texture and crumb porosity of the cake.

UNITERMS: Cupuassu. Kefir. Cake. Image analysis. Texture. Food color

\section{REFERÊNCIAS BIBLIOGRÁFICAS}

BAIK, O.D.; MARCOTE, M.; CASTAIGNE, F. Cake baking in tunnel type multi-zone industrial ovens. Part II. Evaluation of quality parameters. Food Res. Int., Amsterdam, v.33, p. 599-607, 2000.

CAUVAIN, S.P. Improving the control of staling in frozen bakery products. Trends in Food Sci Technol., Amsterdam, v.9, n.2, p. 56-61, 1998.

ESTELLER, M.S.; AMARAL, R.L.; LANNES, S.C.S. Effect of Sugar and Fat Replacers on the Texture of Baked Goods. J. Texture Studies, Trumbull, v. 35, p. 383393, 2004a.

ESTELLER, M.S.; YOSHIMOTO, R.M.O .; AMARAL, R.L.; LANNES, S.C.S. Uso de açúcares em produtos panificados. Cienc. Tecnol. de Alim., Campinas, v.24, n.4, p. 602-607, 2004b.

ESTELLER, M.S.; LANNES, S.C.S. Parâmetros Complementares para Fixação de Identidade e Qualidade de Produtos Panificados. Cienc. Tecnol. Alim., Campinas, v.25, n.4, p. 802-806, 2005.

ESTELLER, M.S.; ZANCANARO JÚNIOR, O.; LANNES, S.C.S. The effect of kefir addition on microstructure parameters and physical properties of porous white bread. Eur. Food Res. Technol., Heidelberg, v.222, p. 26$31,2006 \mathrm{a}$.

ESTELLER,M.S.; LIMA, A.C.O.; LANNES, S.C.S. Color measurement in hamburger buns with fat and sugar replacers. LWT, Wädenswil,v.39, p. 184-187, 2006 b.

FARNWORTH, E. R. Handbook of fermented functional foods. Boca Raton: CRC Press, 2003. 390p.

GIESE, J. Color measurement in foods as a quality parameter. Food Technol., Chicago, v.54, n.2, p.62-63, 2000.

JOOSTE, M.E. Cake structure and palability as affected by emulsifying agents and baking temperatures. Corvallis, 1951. Ph.D. Thesis. Oregon State College. 
LABUZA, T.P.; HYMAN, C.R. Moisture migration and control in multi-domain foods. Trends Food Sci. Technol., Amsterdam, v.9, p.47-55, 1998

LANNES, S.C.S.; MEDEIROS, M.L. Processamento de achocolatado de cupuaçu por spray-drier. Rev. Bras. Cienc. Farm., São Paulo, v. 39, n.1, p.115-123, 2003.
PLESSAS, S.; PHERSON, L.; BEKATOROU, A. A.; NIGAN, P.; KOUTINAS, A.A. Bread making using kefir grains as baker's yeast. Food Chem., Amsterdam, v.93, n.4, p.585-589, 2005.

Recebido para publicação em 05 de outubro de 2005. Aceito para publicação em 26 de abril de 2006. 